\title{
Risk Markers for Depression in Adolescents: Sleep and HPA Measures
}

\author{
Uma Rao*, , Constance L Hammen ${ }^{2}$ and Russell E Poland ${ }^{3}$ \\ 'Department of Psychiatry, the University of Texas Southwestern Medical Center, Dallas, TX, USA; ${ }^{2}$ Department of Psychology, the University of \\ California, Los Angeles, CA, USA; ${ }^{3}$ The Research and Education Institute for Texas Health Resources, Arlington, TX, USA
}

\begin{abstract}
Previous work has demonstrated reliable electroencephalographic (EEG) sleep and hypothalamic-pituitary-adrenal (HPA) changes associated with adult major depressive disorder. These changes might be evident before clinical manifestation of the illness in at-risk persons. The aim of the study was to identify depression-related EEG sleep and HPA changes in healthy adolescents at high risk for depression, and to examine the relationship between EEG sleep (or HPA) changes and the onset of depression. Forty-eight adolescent volunteers with no personal history of a psychiatric illness, including depression, but who were at high risk for developing depression by virtue of parental depression (high-risk group), and 48 adolescent volunteers with no personal or family history of a psychiatric disorder (normal controls) were recruited. EEG sleep and HPA measures were collected on three consecutive evenings and nights at baseline. Clinical follow-up evaluations were conducted at regular intervals over a 5-year period. Compared with normal controls, adolescents at high risk for depression had shorter latency to rapid eye movement (REM) sleep, increased phasic REM sleep, more REM sleep and elevated nocturnal urinary-free cortisol (NUFC) excretion at baseline. Shorter REM latency, higher REM density and elevated NUFC (measured at baseline) were associated with the development of depression during follow-up. The findings that REM sleep abnormalities and elevated HPA activity occur before the onset of depression in at-risk adolescents suggest that these variables serve as vulnerability markers for the illness.

Neuropsychopharmacology (2009) 34, 1936-1945; doi: I0.1038/npp.2009.27; published online 4 March 2009
\end{abstract}

Keywords: cortisol; depression; development; EEG sleep; pediatric; vulnerability

\section{INTRODUCTION}

Adolescence is the highest risk period for the development of major depressive disorder, and there is evidence of an increasing secular trend (Kessler et al, 2001). There also are data showing that early depressive episodes recur or persist into adult life along with ongoing psychosocial difficulties, including disruption in interpersonal relationships, early pregnancy, low educational attainment, poor occupational functioning and unemployment, as well as elevated risk of suicidal behavior (Rao and Chen, 2009). Given the growing economic and psychosocial burden associated with juvenile depression, the identification of youth at highest risk of illness will have great public health impact (Rao, 2006).

Although genetic transmission of major depressive disorder has been well documented, efforts to identify disease loci have not been successful, most likely because depression is a complex disorder with a varying collection

*Correspondence: $\operatorname{Dr} \cup$ Rao, Department of Psychiatry, The University of Texas Southwestern Medical Center, 5323 Harry Hines Boulevard, Mail Box 9I0I, Dallas, TX 75390-9I0I, USA,

Tel: + 214648 5288, Fax: + 2146485242 ,

E-mail: uma.rao@utsouthwestern.edu

Received 4 October 2008; revised 3 February 2009; accepted 5 February 2009 of clinical phenotypes and polygenic origins (Eaves et al, 2003; Kendler et al, 2001; Uher, 2008). A geneticallymediated physiological measure, an 'endophenotype,' can be helpful in reducing the heterogeneity (Gottesman and Gould, 2003). Such a marker can simplify phenotypes, identify the mode of inheritance and potentially increase detection of at-risk individuals. On the basis of the evidence of a strong genetic influence on sleep architecture (Kimura and Winkelmann, 2007), and on the basis of the welldescribed sleep disruptions associated with depressive disorder (American Psychiatric Association, 1994; Benca et al, 1992; Breslau et al, 1996; Buysse et al, 2008), sleep measures can serve these functions.

Prospective epidemiological studies demonstrated that sleep disturbances increase the risk for depressive disorders (Breslau et al, 1996; Buysse et al, 2008). Although no single sleep marker is specifically associated with major depressive disorder, a constellation of sleep changes has been observed consistently in the laboratory through electroencephalographic (EEG) sleep measures, in adult patients (Benca et al, 1992; Germain and Kupfer, 2008; Tsuno et al, 2005). The most reliable EEG sleep changes associated with major depression include sleep continuity disturbances, shorter latency to rapid eye movement (REM) sleep, increased phasic REM sleep, and diminished slow-wave sleep. 
In contrast to the data in adults, EEG sleep measures have shown considerable variability with regard to group differences between depressed youngsters and matched controls (Ivanenko and Johnson, 2008; Kaufman et al, 2001). The findings vary as a function of age, gender, familial risk for depression, severity of illness, and clinical course (Kaufman et al, 2001; Robert et al, 2006). There is evidence that short REM latency and related sleep characteristics are familial and they might be associated with increased risk for depression, beyond the risk conferred by depressive illness in relatives (Giles et al, 1987, 1998; Morehouse et al, 2002; Rao et al, 1996). The EEG sleep disruptions associated with depressive illness are remarkably stable during recovery and across multiple episodes (Giles et al, 1993; Poland et al, 1997; Rao and Poland, 2008; Thase et al, 1995).

In addition to sleep, there has been considerable interest in the hypothalamic-pituitary-adrenal (HPA) system, consistent with the hypothesis that depression is linked to altered responses to stress. Other lines of evidence suggest that corticotropin-releasing hormone $(\mathrm{CRH})$ and glucocorticoids modulate the regulation of sleep (Schmid et al, 2008; Steiger, 2007). Numerous studies documented HPA dysregulation in adult depression including higher basal $\mathrm{CRH}$ and cortisol secretion, non-suppression of cortisol in response to dexamethasone administration, and blunted corticotropin response to $\mathrm{CRH}$ administration (Holsboer, 2001; Young, 2004). Also, several studies reported that REM latency correlates with nocturnal cortisol secretion during the depressive episode, suggesting that dysregulation of mood, sleep, and HPA axis might be linked, at least in part, by common neuronal systems (Asnis et al, 1983; Poland et al, 1992; Rao et al, 1996; Rao and Poland, 2008).

Findings from HPA studies in depressed children and adolescents have been inconsistent (Kaufman et al, 2001; Zalsman et al, 2006). In particular, depressed children did not display changes in 24-h cortisol patterns. Few differences in basal cortisol secretion have been observed between depressed adolescents and controls, and when group differences were detected, they tend to be subtle alterations in normal diurnal patterns. These subtle changes, however, were relatively robust in predicting the longitudinal clinical course; higher cortisol secretion in the evening or during sleep, a time when the HPA axis is relatively quiescent, was associated with a longer time to recovery from the depressive episode (Goodyer et al, 2001), a propensity for recurrence (Rao et al, 1996), and suicide attempts (Mathew et al, 2003). HPA activity also is heritable (Kirschbaum et al, 1992; Meikle et al, 1988; Ouellet-Morin et al, 2008), and higher cortisol secretion was detected in atrisk youth who subsequently developed depression (Goodyer et al, 2000; Halligan et al, 2007).

This study was undertaken to replicate and extend earlier observations on the associations among family/genetic factors, EEG sleep manifestations, HPA dysregulation, and vulnerability to depression. It was hypothesized that healthy adolescents (with no earlier psychiatric history) who are at high-risk for developing depression by virtue of depression history in the parent will manifest depression-related EEG sleep and HPA changes compared with normal controls. Also, it was hypothesized that the observed sleep and HPA changes at baseline will predict the development of a depressive episode during prospective follow-up above and beyond the effects of parental depression. It was expected that the sleep and HPA 'vulnerability markers' for depression are not specific to the high-risk status (familial depression), but they occur at a higher frequency in this group of youngsters.

\section{MATERIALS AND METHODS}

\section{Participants}

The protocol was approved by the Institutional Review Boards at the University of California at Los Angeles and affiliated institutions. All adolescents signed a written assent form, and parents signed an informed consent document before performing the research procedures. The participants were recruited from local pediatric and mental health clinics and schools in Los Angeles, through advertisements in local newspapers, and by word-of-mouth. The sample included 48 adolescents with no lifetime personal or family history of psychiatric illness, and 48 adolescents with no personal history of a psychiatric disorder, including depression, but were at high risk for developing depression by virtue of parental depression; at least one biological parent with a history of unipolar major depressive disorder who required treatment. All participants were medically healthy and free from alcohol or illicit drug use, as determined by physical examination, full chemistry panel, thyroid function tests, and electrocardiogram and urine drug screens. Subjects with a personal history of sleep disorder(s), or those with a family history of narcolepsy, and females with suspected pregnancy were excluded from the study.

\section{Assessments}

Diagnostic evaluation. Symptoms of major psychiatric disorders were assessed using the Schedule for Affective Disorders and Schizophrenia for School-Age Children - the Present and Lifetime Version (K-SADS-PL). The K-SADS$\mathrm{PL}$ is a semi-structured interview designed to ascertain present and lifetime history of psychiatric illness according to the DSM-IV criteria (Kaufman et al, 1997). Probes and objective criteria are provided for individual symptoms at both diagnostic and sub-threshold levels. Inter-rater and test-retest reliability have been established, as well as convergent and discriminate validity (Kaufman et al, 1997). The K-SADS-PL was administered separately to the parent and the adolescent, and both were re-interviewed to resolve any discrepancies. The summary scores were tabulated based on the information obtained from both informants. The Hamilton Depression Rating Scale (HDRS; Hamilton, 1960), a depression severity measure, and Children's Global Assessment Scale (CGAS; Shaffer et al, 1983), a global psychosocial functioning measure, also were completed. The adolescent participants completed the Beck Depression Inventory (BDI) for self-assessment of depression severity (Beck et al, 1961).

The Family History-Research Diagnostic Criteria (FHRDC), a semi-structured interview, was used for the evaluation of psychiatric disorders in family members (Andreasen et al, 1977). A parent was interviewed regarding 
lifetime psychiatric disorders in all the first-degree relatives of the adolescent subject (including the self, spouse, and all offspring). The FH-RDC is sensitive for obtaining information from knowledgeable relatives (Thompson et al, 1982). Information on race/ethnicity was gathered from selfreport, and socioeconomic status (SES) was assessed with the Hollingshead Scale (Hollingshead, 1975).

\section{Regulation of sleep-wake schedule}

One week before the laboratory assessment, the participants were instructed to go to bed between 2200 and 2300 hours, and to awake between 0630 and 0730 hours. The sleep-wake schedules were monitored by daily sleep logs and wrist actigraphy.

\section{Sleep and neuroendocrine protocol}

The protocol comprised a 3-night study. In order not to cause potential disruption in sleep because of catheter insertion and blood draws, as a reflection of the HPA activity, salivary cortisol before sleep, and nocturnal urinary-free cortisol (NUFC) measures were collected. The participants arrived in the laboratory at 2000 hours, and saliva samples were collected at half-hour intervals from 2030 until 2130 hours. Conventional EEG electrodes were attached by 2100 hours, and sleep recordings were taken from 2230 (lights out) to 0700 hours. Subjects were asked to void urine at 2230 hours before switching off the lights. All urine voided between 2230 and 0700 hours (including the 0700 hours sample) was collected. To rule out the presence of major sleep disorders, a full sleep polysomnography was performed on the first night, including respiratory, oximetry, and leg movement measurements. The International 10-20 System was used for EEG electrode placement, electromyogram (EMG), electrooculogram (EOG), and electrocardiogram. Bilateral EEG recordings were obtained from the left (C3) and right (C4) central leads, referenced to the opposite mastoid, A2 and A1, as well as to a linked reference $(\mathrm{A} 1+\mathrm{A} 2)$. Bilateral EOG recordings were obtained, and referenced to $\mathrm{A} 1+\mathrm{A} 2$ along with a submental EMG recording.

\section{Follow-up evaluation}

After the baseline assessments, the participants were followed longitudinally at 6-month intervals in the first year and yearly thereafter, for up to 5 years, to obtain information on the development of depression and other psychiatric disorders. In addition to obtaining information from the K-SADS-PL during each follow-up evaluation, the Longitudinal Interval Follow-up Evaluation (LIFE) was used to document the onset and clinical course of depression. The LIFE is a semi-structured instrument used for charting the clinical course of depression and other psychiatric disorders during longitudinal follow-up (Shapiro and Keller, 1979). The development of a depressive episode was defined as a rating of $\geqslant 5$ on the Psychiatric Status Rating (PSR) component of the LIFE for a minimum of 4-week duration. The 4-week duration was used to ensure stability of symptoms as depressive symptoms in youngsters tend to be more variable, with greater heterogeneity in clinical response and course, than adults. The PSR is a 6-point scale providing information on the severity of depressive symptomatology. Information from the clinical follow-up assessments was presented to an independent person 'blind' to the group and psychobiological status, and the final diagnosis was based on consensus ratings.

\section{Scoring of sleep records and cortisol assays}

Sleep records were coded and scored 'blindly' according to the standard criteria (Rechtschaffen and Kales, 1968). REM latency was defined as the time between sleep onset (first minute of stage 2 or deeper sleep, followed by at least $9 \mathrm{~min}$ of stage 2 or deeper sleep, interrupted by no more than $1 \mathrm{~min}$ of waking or stage 1) and the first REM period $\geqslant 3 \mathrm{~min}$ in length. Other REM sleep measures, including REM activity and REM density, and additional sleep variables were also scored according to the standard criteria (Kupfer et al, 1976). REM activity was scored on a scale ranging from 0 to 8 units.

Salivary cortisol and NUFC concentration were assayed using the radioimmunoassay method, as described earlier (Poland and Rubin, 1982; Rao et al, 1997). The intra- and inter-assay coefficients of variation for the assays were less than $10 \%$. Samples from the same subject were analyzed in the same assay.

\section{Statistical Methods}

For all summary variables, data were examined for normality using the Shapiro-Wilk's W statistic (Shapiro and Wilk, 1965). In cases of significantly non-normal distributions, logarithm transformations were performed to normalize the data before the application of statistical tests for significance. Student's $t$-test and Pearson's $\chi^{2}$-test were used for group comparisons on demographic and clinical variables. Multivariate analysis of covariance (MANCOVA) was used for comparisons on categories of EEG sleep and HPA measures, controlling for demographic and clinical variables that were significantly different between groups. Group comparisons of individual means were tested if the overall MANCOVA was significant. Regression procedures were used for evaluating the associations between group status (baseline or follow-up diagnostic status) and sleep (or HPA) measures that were significant in the MANCOVA tests. Effect sizes were calculated, using mean difference and standard deviation for continuous variables and odds ratio for categorical measures. The first night was considered as an adaptation night, and the mean values derived from Night 2 and Night 3 data were used. For salivary cortisol, mean values of the three samples on Night 2 and Night 3 were taken. $\alpha$ was set at 0.05 .

\section{Follow-up Information}

Three controls and four high-risk subjects did not have any follow-up evaluations. Subjects who did not participate in the follow-up assessments did not differ significantly from those with follow-up information on any demographic or clinical characteristics. Recruitment did not occur 
simultaneously and, therefore, not all subjects were studied longitudinally for the same period of time. Of the 89 adolescents who had follow-up information, $7.9 \%$ were followed for 2 years, $22.4 \%$ for 3 years, $33.7 \%$ for 4 years, and $36.0 \%$ for 5 years. The groups were comparable on the mean follow-up interval (mean follow-up interval $=3.6$ years; median $=3.6$ ).

\section{RESULTS}

\section{Demographic and Clinical Characteristics of the Sample}

Demographic and clinical features at recruitment are outlined in Table 1. Adolescents at high risk for depression had significantly lower SES, higher BDI, and lower CGAS scores compared with normal controls. Despite the statistical difference on SES scores, the mean value in both groups was in the second highest category of social class and the participants came primarily from the middle and upper middle classes (100\% of normal controls, and $87.5 \%$ of high-risk subjects). Despite the statistical differences, the high-risk group did not express clinically significant depressive symptoms or functional impairment, with scores within the normal range (see Table 1). Within the high-risk group, 23 subjects $(47.9 \%)$ had only a maternal history, 10 (20.8\%) had only a paternal history, and $15(31.3 \%)$ had depression history in both parents. Twenty parents (41.6\%) experienced the first episode of depression before the age of 21 years, and $34(70.8 \%)$ had recurrent episodes.

\section{EEG Sleep and HPA Measures at Baseline}

Mean values for the major EEG sleep and HPA variables for both groups are shown in Table 2. After controlling for the effects of SES, depression severity (BDI), and functional (CGAS) measures, the high-risk subjects had significantly shorter REM latency, higher proportion of REM sleep, increased REM activity and REM density, longer REM duration, more number of REM episodes, as well as elevated NUFC excretion compared with controls. Post hoc tests showed that there was a tendency for high-risk youth with depression history in both parents to have shorter REM latency $\left(80.2 \pm 34.5\right.$ vs $97.6 \pm 38.9, \mathrm{t}_{46}=1.76, \quad p=0.09$, $\mathrm{ES}=-0.49, \quad \mathrm{CI}=-1.11-0.13)$ and higher REM density $\left(2.7 \pm 0.6 \quad\right.$ vs $2.4 \pm 0.5, \quad \mathrm{t}_{46}=1.72, \quad p=0.10, \quad \mathrm{ES}=0 . \quad 56$, $\mathrm{CI}=0.25-0.76)$ compared with those with depression history in one parent only.

To test the relative contribution of EEG sleep and HPA measures to the prediction of group membership (control $v s$ high-risk status), logistic regression analyses were conducted. Demographic (age, gender, ethnicity, and SES) and clinical (BDI, HDRS, and CGAS) variables were entered in the first step in these analyses $\left(\chi^{2}=21.91, \mathrm{df}=7, p=0.005\right)$. After controlling for these effects, sleep and HPA measures predicted group status $\left(\chi^{2}=26.85, \mathrm{df}=7, p=0.0001\right)$. Together, the sleep and HPA measures accurately identified $35 / 48(72.9 \%)$ normal controls and 33/48 (68.8\%) high-risk subjects. However, there was a significant correlation among several measures (see Table 3 ). Only REM latency $(\mathrm{OR}=0.15, \mathrm{CI}=0.03-0.85, p=0.04)$ had significant predictive power in discriminating the two groups. NUFC showed a nonsignificant trend in predicting group status $(\mathrm{OR}=1.05, \mathrm{CI}=1.00-1.11, p=0.10)$.

An alternative approach was taken for graphical representation of the frequency of 'abnormal' sleep markers. Using a receiver operator characteristic (ROC) analysis, Giles et al (1990b) reported age-adjusted threshold values for short REM latency in normal and depressed populations. A mean REM latency value of $\leqslant 70 \mathrm{~min}$ was considered abnormal for the younger patients (Giles et al, $1990 \mathrm{~b})$. On the basis of this criterion, $2 / 48(4.1 \%)$ normal controls, and 15/48 (31.3\%) high-risk adolescents manifested short REM latency $\left(\chi^{2}=12.08, p=.001, \mathrm{OR}=0.10\right.$, $\mathrm{CI}=0.20-0.45$; see Figure 1).

\section{Relationship Between Parental Depression and Development of Adolescent Depression During Prospective Follow-Up}

Among 45 controls who had follow-up information, four (8.9\%) developed a depressive episode during the study period, whereas $10 / 44(22.7 \%)$ of high-risk subjects developed the disorder $\left(\chi^{2}=3.21, p=0.07, \mathrm{OR}=0.33, \mathrm{CI}=0.10\right.$ $1.15)$. Of a total of 14 youth who developed a depressive episode during follow-up, the mean time to depressive episode from intake was 2.3 years $(S D=0.9$ years; range $=0.7-4.1$ years). Within the high-risk group, familial loading (depression in one parent $v s$ both parents) was not

Table I Baseline demographic and clinical parameters in controls and high-risk adolescents

\begin{tabular}{lcccc}
\hline & Control $(\boldsymbol{n}=\mathbf{4 8})$ & High-risk $(\boldsymbol{n}=\mathbf{4 8})$ & Statistic & E-value \\
\hline Age (years) & $15.2 \pm 1.4$ & $15.0 \pm 1.5$ & 0.49 & 0.51 \\
Gender (male/female) & $19 / 29$ & $21 / 27$ & 0.17 & 0.68 \\
Ethnicity (AA/AS/CC/HS) & $6 / 10 / 23 / 9$ & $7 / 8 / 23 / 10$ & 0.35 & 0.14 \\
Socioeconomic score ${ }^{a}$ & $49.1 \pm 9.4$ & $43.5 \pm 10.1$ & 2.80 & 0.95 \\
HDRS & $0.9 \pm 1.4$ & $1.0 \pm 1.4$ & 0.36 & 0.006 \\
BECK DEPRESSION INVENTORY & $1.7 \pm 2.4$ & $3.5 \pm 3.1$ & 3.06 & 0.72 \\
CGAS & $83.2 \pm 9.7$ & $78.9 \pm 10.0$ & 2.16 & 0.003 \\
\hline
\end{tabular}

Data are presented in means and SDs, or in raw numbers.

AA, African-American; AS, Asian-American; CC, Caucasian; CGAS, Children's Global Assessment Scale; ES, effect size; HS, Hispanic; HDRS, Hamilton Depression Rating Scale.

aHigher score is associated with higher socioeconomic status (Hollingshead Scale). 
Table 2 EEG sleep and HPA variables (mean \pm SD) in control and high-risk adolescents ${ }^{\mathrm{a}}$

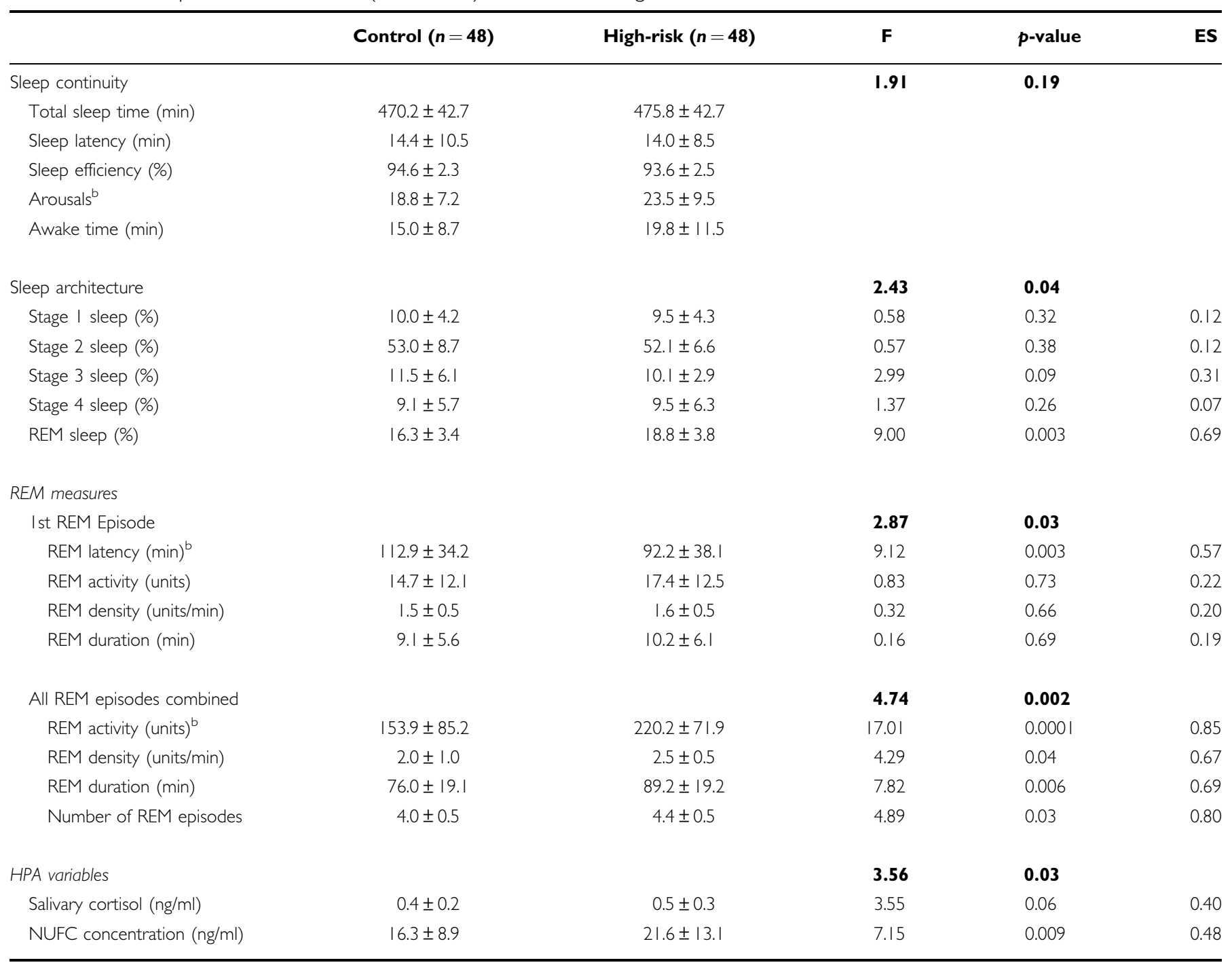

ES, effect size.

aDepression severity, and socioeconomic and functional status were included as co-variates.

${ }^{b}$ Analyses were performed on log-transformed data.

Table 3 Correlations among EEG sleep and HPA variables that discriminated control and high-risk adolescents

\begin{tabular}{|c|c|c|c|c|c|c|c|}
\hline & REM sleep (\%) & REM latency & REM activity & REM density & REM duration & REM episodes & NUFC \\
\hline REM sleep (\%) & 1.00 & -0.09 & $0.58 * *$ & 0.12 & $0.91 * *$ & $0.80 * *$ & 0.14 \\
\hline REM latency & - & 1.00 & -0.11 & -0.11 & -0.06 & -0.07 & -0.16 \\
\hline REM activity & - & - & 1.00 & $0.72 * *$ & 0.60** & $0.50 * *$ & $0.21 *$ \\
\hline REM density & - & - & - & 1.00 & 0.05 & -0.00 & 0.02 \\
\hline REM duration & - & - & - & - & 1.00 & $0.91 * *$ & $0.23 *$ \\
\hline
\end{tabular}

* $p \leqslant 0.05 ; * * * \leqslant 0.01$.

associated with vulnerability to depression. Of a total of 31 high-risk youth with depression history in one parent, five (16.1\%) developed a major depressive episode during follow-up, whereas 5/13 (38.5\%) adolescents with depression history in both parents developed the illness (Fisher's Exact Test, $p=0.13, \mathrm{OR}=0.31, \mathrm{CI}=0.07-1.34$ ). 
Association Between Baseline Neurobiological Measures and Vulnerability to Depression

Specific EEG sleep and HPA measures that discriminated controls and high-risk subjects in the original analyses (see Table 2) were selected for evaluating whether these measures predicted a depressive episode during follow-up (see Table 4). In the initial step, the predictive value of

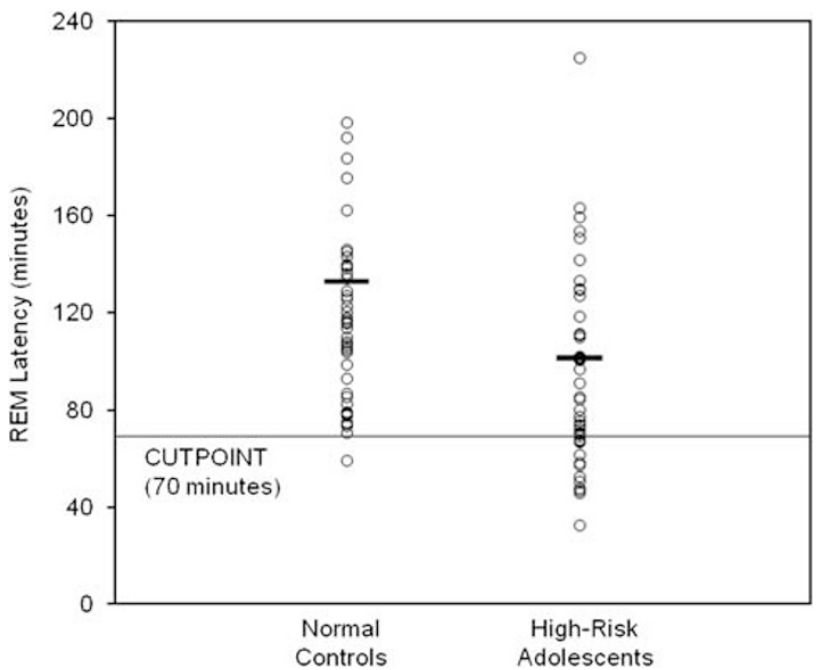

Figure I REM latency values (mean of Night 2 and Night 3 ) in individual subjects within normal control $(n=48)$ and high-risk $(n=48)$ groups. The horizontal lines (within each column of circles) represent mean values for the two groups. A cut point of 70 min was considered as abnormal value. demographic and clinical variables was evaluated. Although the regression model was not significant, group status (control vs high-risk subjects) and HDRS score were significant predictors of depression (see Block 1 in Table 4). The demographic and clinical variables identified 74/75 (98.7\%) adolescents without depression, but only 2/14 (14.3\%) of those with depression. After controlling for these factors, the sleep and HPA measures predicted depression during follow-up (see Block 2 in Table 4). Specifically, subjects who developed depression had shorter REM latency, higher REM density, and more NUFC excretion compared to their counterparts who did not develop depression. The neurobiological measures accurately identified 74/75 (98.7\%) of subjects without depression and 8/14 (57.1\%) adolescents with depression.

As described above, a REM latency value of $\leqslant 70 \mathrm{~min}$ was considered abnormal (Giles et al, 1990b), and the association between REM latency and vulnerability to depression was assessed. Of two normal controls with short REM latency, one (50.0\%) developed a depressive episode, whereas $3 / 43$ (7.0\%) subjects with normal REM latency developed the illness during follow-up (Fisher's Exact Test, $p=0.17, \mathrm{OR}=13.33, \mathrm{CI}=0.66-270.47)$. Within the high-risk group, 6/14 (42.9\%) with short REM latency developed a depressive episode in comparison with 4/30 (13.3\%) youngsters with normal REM latency (Fisher's Exact Test, $p=0.05, \quad \mathrm{OR}=4.88, \quad \mathrm{CI}=1.10-21.69)$. No normative data are available with respect to cortisol measures. Using the criterion of $\geqslant 1$ standard deviation of mean NUFC concentration in normal controls as elevated

Table 4 Hierarchical regression equations predicting major depressive disorder at follow-up

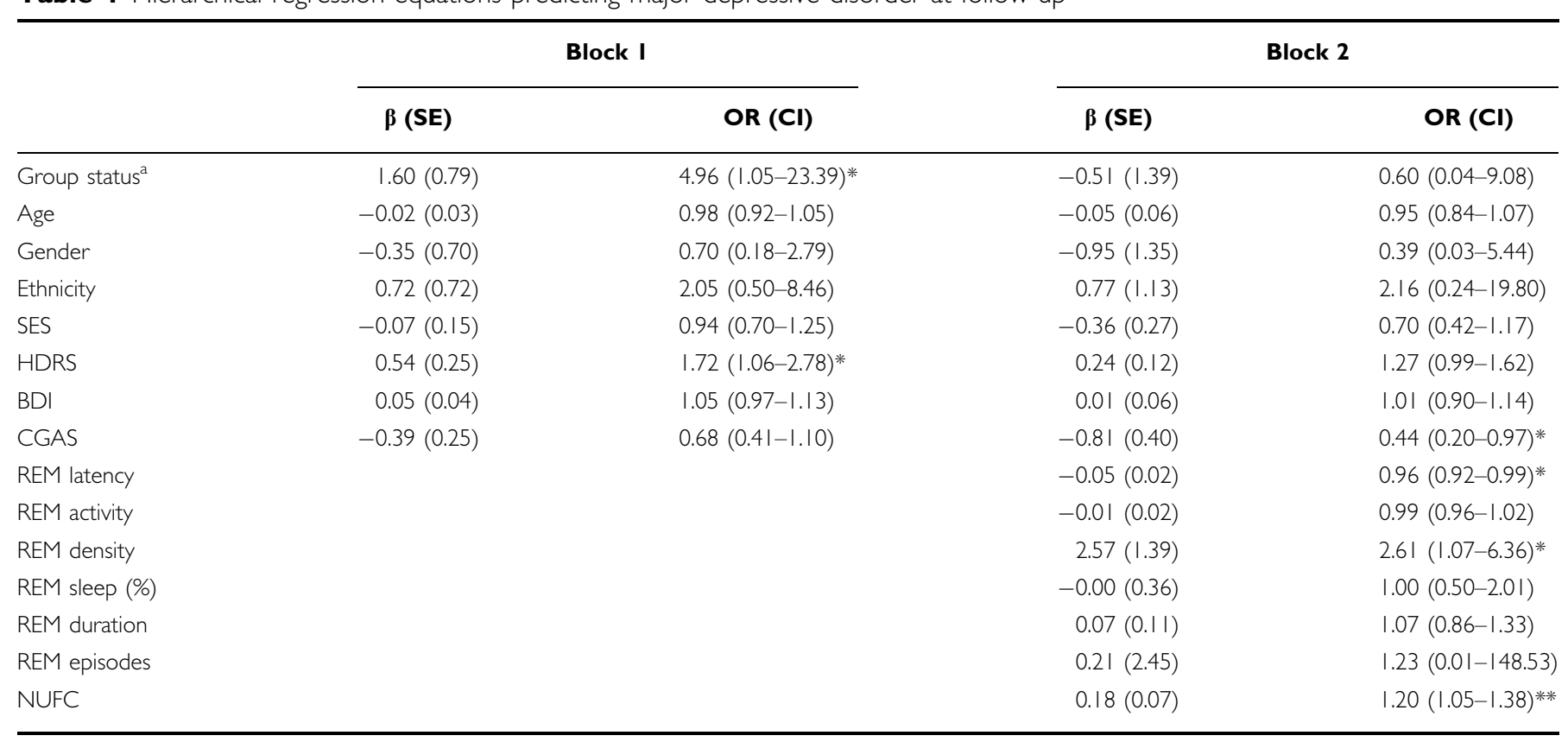

BDI, Beck Depression Inventory; CGAS, Children's Global Assessment Scale; Cl, confidence interval; HDRS, Hamilton Depression Rating Scale; OR, odds-ratio; REM, rapid eye movement; SES, socioeconomic status; SE, standard error.

$* 0 \leqslant 0.05 ; * * * \leqslant 0.01$.

Block I: overall $\chi^{2}=11.98, \mathrm{df}=8, p=0.10 ; \delta \chi^{2}=11.98, \mathrm{df}=8, p=0.10$.

Block 2: overall $\chi^{2}=42.66, \mathrm{df}=15, p=0.000 \mathrm{I} ; \delta \chi^{2}=30.68, \mathrm{df}=7, p=0.0001$

anitial group status $=$ normal, and high-risk (normal controls served as the reference group). 
HPA activity, $2 / 6(33.3 \%)$ controls with elevated HPA activity, and $2 / 39(5.1 \%)$ with normal HPA activity developed a depressive episode (Fisher's Exact Test, $p=0.08, \quad \mathrm{OR}=9.25, \quad \mathrm{CI}=1.01-84.73)$. Among high-risk subjects, $7 / 18$ (38.9\%) with elevated HPA activity developed depression in contrast to $3 / 26(11.5 \%)$ youngsters with normal HPA activity (Fisher's Exact Test, $p=0.06$, $\mathrm{OR}=4.88, \mathrm{CI}=1.05-22.57$ ).

\section{DISCUSSION}

The findings from the study indicated that adolescents with familial risk for depression, but without manifestation of a clinical diagnosis, showed evidence of EEG sleep and/or HPA changes that were typically reported in the literature as characteristic of a depressive episode. Also, the observed changes in EEG sleep and HPA measures at baseline (during recruitment) were associated with the development of depression during prospective follow-up, suggesting that they are vulnerability markers for depression. These 'risk markers' contributed to the prediction of depression above and beyond the contribution made by familial risk for depression, as well as by relevant clinical and demographic variables. It is important to note that the sleep and HPA markers are not specific to the high-risk status and that they are also observed in youth without known familial/genetic risk for depression. However, they appear to occur more frequently in the high-risk group and, therefore, the EEG sleep and HPA markers might be helpful in identifying 'atrisk' individuals who may have the highest risk for depressive illness.

These results should be considered preliminary because of the modest sample sizes, with inadequate power to detect potential group differences. Also, because of the relatively short follow-up interval, some youth who manifested EEG sleep and/or HPA abnormalities did not develop depression, thereby precluding our ability to estimate the full magnitude of risk associated with these markers. More stringent criteria were used for the diagnosis of depression (a 4-week duration). Although this method identified individuals with more sustained depressive symptoms, some cases with a depressive disorder could have been misclassified. In addition to manifesting sleep and HPA changes at a greater frequency, the high-risk group also had higher level of depressive symptoms and lower level of psychosocial functioning and SES than normal controls. Although subsyndromal depressive symptoms and lower functional and SES are known risk factors for depression (Joiner, 2006), the sleep and HPA measures made unique contributions. Moreover, the first depressive episode occurred 7 months after the initial clinical and neurobiological assessments, suggesting a trait-like nature of the neurobiological measures. Depressive symptoms and functional measures were gathered during each follow-up evaluation, but these variables did not always coincide with the development of a depressive episode. The onset of a depressive episode was documented retrospectively encompassing the entire follow-up interval, whereas the depression severity (BDI and HDRS) and functional (CGAS) measures reflected only a 2 -week period preceding the follow-up evaluation.
Despite the limitations described above, the study findings are consistent with earlier reports in children, adolescents, and adults. Coble et al (1988) found higher REM counts in neonates whose mothers had a history of depression compared with their counterparts without maternal depression. In a second study, adolescent females with maternal history of depression manifested low intrahemispheric temporal coherence of EEG sleep rhythms, although no macroarchitectural changes were detected in this sample (Morehouse et al, 2002). On the basis of ROC analysis, $54 \%$ of high-risk girls were identified with extreme low coherence (Morehouse et al, 2002). Exposure to maternal depression during the postnatal period was associated with higher and more variable cortisol secretion in adolescents compared with their peers without exposure to maternal depression (Halligan et al, 2004). Other investigators reported depression-related sleep macro- or microarchitectural changes (Friess et al, 2008a; Fulton et al, 2000; Giles et al, 1998; Lauer et al, 1995), abnormal REM induction in response to a cholinergic challenge (Schreiber et al, 1992; Sitaram et al, 1987), and altered HPA regulation (Friess et al, 2008b; Mannie et al, 2007) in some unaffected adults whose first-degree relatives had a history of depressive disorder.

There was a tendency for 'unaffected' adolescents with both maternal and paternal depression history to have shorter REM latency and higher REM density compared with those with depression history in one parent only. Sleep measures were not obtained in the parents and, consequently, it is not known whether the REM sleep abnormalities are transmitted to the offspring from one or both parents along with the vulnerability for depression. In a preliminary investigation, Giles et al (1992) measured EEG sleep in parents and offspring from 14 families, and found that the sleep patterns of parents and offspring were highly correlated when there was a family history of major depressive disorder. When family history of depression co-occurred with abnormal sleep in the parent, the association between parent and offspring sleep was even stronger, suggesting that vulnerability for depressive illness and abnormal EEG sleep patterns co-segregate in families (Giles et al, 1987, 1992, 1998).

The results of this study also showed that the observed EEG sleep and HPA abnormalities in adolescents, who had no earlier psychiatric history at the time of assessment, were associated with increased vulnerability for depression during prospective follow-up. In a earlier investigation, we reported that $37 \%$ of adolescents that manifested short REM latency developed depression during a 7-year period, whereas only $7 \%$ of youngsters with normal REM latency developed the illness (Rao et al, 1996). In addition, $67 \%$ of adolescents with elevated nocturnal cortisol secretion developed depressive illness compared with $12 \%$ of individuals with normal HPA activity in that study. Similarly, $41 \%$ of adolescent females identified as having abnormal temporal coherence and high familial risk for depression manifested depressive symptoms, or developed a depressive episode, within a 2-year period (Morehouse et al, 2002). Elevated HPA activity in unaffected adolescents that were at high-risk for depression predicted depression during prospective follow-up, and the contribution of elevated HPA activity to depression vulnerability was over 
and above the effects of depressive symptoms at baseline and intervening environmental factors (Goodyer et al, 2000; Halligan et al, 2007). The occurrence of EEG sleep and/or HPA abnormalities in unaffected youth that are at familial risk for depression suggest that they might be useful in identifying those at highest risk for developing the illness.

In adult samples with prospective data, Giles and Kupfer (1994) reported that short REM latency doubled the risk for depression in unaffected adult family members of depressed probands. The presence of high REM density in unaffected high-risk individuals predicted an 'affective spectrum disorder' in another study (Modell et al, 2005). Also, significant shortening of REM latency in response to a cholinergic agonist predicted onset of depression in subjects with family history of depression (Lauer et al, 2004). However, baseline EEG sleep measures did not show predictive power in this high-risk sample, although many individuals who developed depression (57\%) also had short REM latency $(\leqslant 60 \mathrm{~min})$ at baseline. With respect to HPA measures, higher mean cortisol level in the morning predicted depression subsequently in women, and the contribution of HPA activity to depression-risk was above and beyond the contribution of adverse life events and other vulnerability factors (Harris et al, 2000). In contrast to these findings, in a different investigation, adrenocorticotropin and cortisol responses to corticotropin-releasing hormone did not discriminate at-risk persons who developed an affective disorder $v s$ those who remained well despite the response patterns remaining stable after 4 years (Ising et al, 2005; Modell et al, 1998).

One possibility for the inconsistent findings among adult studies, and between adult and adolescent samples, is that many adult subjects would have passed through the risk period for developing depression at the time of initial recruitment, thus yielding only a modest number of persons with the disorder. For example, in an adult sample reporting on EEG sleep and HPA measures, approximately $25 \%$ of individuals with familial risk developed an affective illness over 10 years (Ising et al, 2005; Modell et al, 2005). Also, there was significant heterogeneity with respect to affective illness in the high-risk sample in that study (including unipolar depression, bipolar disorder and 'affective spectrum disorders' comprising of other depressive disorders, adjustment disorder, somatoform disorder, anxiety disorders, and obsessive-compulsive disorder), as well as in the relatives including unipolar, bipolar, and/or schizoaffective disorder (Carroll, 2007). In contrast, Giles et al (1998) recruited only probands with unipolar depression and stratified them on short and normal REM latency, and the reported outcome in family members was major depressive disorder (Giles and Kupfer, 1994).

In addition to identifying highly vulnerable persons, familial aggregation of specific sleep and HPA abnormalities might delineate a valid subtype of the depressive illness. For instance, familial aggregation of short REM latency was associated with similar clinical features and psychological profiles of depression among relatives (Giles et al, 1988, 1990a). Despite the association between specific EEG sleep and HPA changes and increased risk for depression in the above-described studies, a significant proportion of individuals with these markers did not (at least yet) develop depression, highlighting the notion that no single physiological measure constitutes a definitive marker for depression. Other genetically-mediated neurobiological variables (Caspi et al, 2003), psychological factors (Dyer and Giles, 1994; Lauer et al, 1997; Perlis et al, 1995), and stressful life experiences (Goodyer et al, 2000; Hammen et al, 2004; Harris et al, 2000), singly or in combination, might influence the onset of depression in some individuals. Therefore, sleep and HPA variables must be considered in the context of these other variables in identifying vulnerability markers for depression. For example, environmental factors might play a more prominent role in conferring risk for depression in individuals and families where short REM latency is less prevalent (Cartwright, 1983; Giles et al, 1989; Monroe et al, 1992). Similarly, sleep measures might be associated more closely with neurovegetative symptoms (Perlis et al, 1997), whereas somatic symptoms might be less prominent in depressive episodes resulting from stressful life experiences (Monroe et al, 2001). These data suggest that integration of neurobiological measures with psychological and environmental variables might be more helpful in separating the different phenotypes of depression (Eaves et al, 2003; Uher, 2008). A careful dissection of the phenotypes related to depressive illness not only will be beneficial for identifying the 'culprit' genes, but also in developing more specific treatment and preventive strategies for this highly morbid disorder (Rao, 2006; Simons et al, 1995).

Reduction in slow-wave sleep, a common feature of depression in adult patients, was not observed in the highrisk group. This finding is consistent with the earlier reports showing no slow-wave deficits in depressed adolescents, and suggest developmental discontinuities in sleep regulation in adolescent $v s$ adult depression (Ivanenko and Johnson, 2008; Kaufman et al, 2001). Understanding the developmental continuities and discontinuities in neurobiological substrates of depression not only will be helpful in developing more effective and safer pharmacological agents for the pediatric population, but also helpful in adult patients who do not respond adequately to the currently available treatments (Rao, 2006; Rush et al, 2003).

\section{ACKNOWLEDGEMENTS}

This work was supported, in part, by Grants DA14037, DA15131, DA17804, DA17805, MH01419, MH62464, and MH68391 from the National Institutes of Health, from the National Alliance for Research on Schizophrenia and Affective Disorders, and by the Sarah $\mathrm{M}$ and Charles E. Seay Endowed Chair in Child Psychiatry at UT Southwestern Medical Center. We also thank Li-Ann Chen, MA, for technical support.

\section{DISCLOSURE/CONFLICT OF INTEREST}

The authors have no financial conflict of interest.

\section{REFERENCES}

American Psychiatric Association (1994). Diagnostic and Statistical Manual of Mental Disorders. American Psychiatric Press: Washington, DC. 
Andreasen NC, Endicott J, Spitzer RL, Winokur G (1977). The family history method using diagnostic criteria. Arch Gen Psychiatry 34: 1229-1235.

Asnis GM, Halbreich U, Sachar EJ, Nathan RS, Ostrow LC, Novacenko H et al (1983). Plasma cortisol secretion and REM period latency in adult endogenous depression. Am J Psychiatry 140: $750-753$.

Beck AT, Ward CH, Mendelson M, Muck M, Erbaugh J (1961). An inventory for measuring depression. Arch Gen Psychiatry 4: 561-571.

Benca RM, Obermeyer WH, Thisted RA, Gillin JC (1992). Sleep and psychiatric disorders: a meta-analysis. Arch Gen Psychiatry 49: 651-668.

Breslau N, Roth T, Rosenthal L, Andreski P (1996). Sleep disturbance and psychiatric disorders: a longitudinal epidemiological study of young adults. Biol Psychiatry 39: 411-418.

Buysse DJ, Angst J, Gama A, Ajdacic V, Eich D, Rossler W (2008). Prevalence, course and comorbidity of insomnia and depression in young adults. Sleep 31: 473-480.

Carroll BJ (2007). Regarding 'the Munich vulnerability study on affective disorders: premorbid polysomnographic profile of affected high-risk probands. Biol Psychiatry 62: 703-704.

Cartwright RD (1983). Rapid eye movement sleep characteristics during and after mood-disturbing events. Arch Gen Psychiatry 40: 197-201.

Caspi a, Sudgen K, Moffitt TE, Taylor A, Craig IW, Harrington H et al (2003). Influence of life stress on depression: moderation by a polymorphism in the 5-HTT gene. Science 301: 386-389.

Coble PA, Scher MS, Reynolds III CF, Day NL, Kupfer DJ (1988). Preliminary findings on the neonatal sleep of offspring of women with and without a prior history of affective disorder. Sleep Res 16: 120.

Dyer JG, Giles DE (1994). Familial influence in unipolar depression: effects of parental cognitions and social adjustment on adult offspring. Compr Psychiatry 35: 290-295.

Eaves L, Silberg J, Erkanli A (2003). Resolving multiple epigenetic pathways to adolescent depression. Child Psychol Psychiatry 44: $1006-1014$

Friess E, Modell S, Brunner H, Tagaya H, Lauer CJ (2008a). The Munich vulnerability study on affective disorders: microstructure of sleep in high-risk subjects. Eur Arch Psychiatry Clin Neurosci 258: 285-291.

Friess E, Schmid D, Modell S, Brunner H, Lauer CJ, Holsboer F et al (2008b). Dex/CRH-test response and sleep in depressed patients and healthy controls with and without vulnerability for affective disorders. J Psychiatr Res 42: 1154-1162.

Fulton MK, Armitage R, Rush AJ (2000). Sleep electroencephalographic coherence abnormalities in individuals at high risk for depression: a pilot study. Biol Psychiatry 47: 618-625.

Germain A, Kupfer DJ (2008). Circadian rhythm disturbances in depression. Hum Pharmacol Clin Exp 23: 571-585.

Giles DE, Biggs MM, Rush AJ, Roffwarg HP (1988). Risk factors in families of unipolar depression, I: psychiatric illness and reduced REM latency. J Affect Disord 14: 51-59.

Giles DE, Etzel BA, Biggs MM (1990a). Risk factors in unipolar depression:II relation between proband REM latency and cognitions of relatives. Psychiatr Res 33: 39-49.

Giles DE, Jarrett RB, Rush AJ, Biggs MM, Roffwarg HP (1993). Prospective assessment of EEG sleep in remitted major depression. Psychiatr Res 46: 269-284.

Giles DE, Kupfer DJ (1994). Reduced REM latency: risk factor for first episode of depression. Sleep Res 23: 197.

Giles DE, Kupfer DJ, Rush AJ, Roffwarg HP (1998). Controlled comparison of electrophysiological sleep in families of probands with unipolar depression. Am J Psychiatry 155: 192-199.

Giles DE, Roffwarg HP, Dahl RE, Kupfer DJ (1992). EEG sleep abnormalities in depressed children: a hypothesis. Psychiatry Res 41: $53-63$.
Giles DE, Roffwarg HP, Kupfer DJ, Rush AJ, Biggs MM, Etzel BA (1989). Secular trend in unipolar depressions: a hypothesis. J Affect Disord 16: 71-75.

Giles DE, Roffwarg HP, Rush AJ (1987). REM latency concordance in depressed family members. Biol Psychiatry 22: 907-910.

Giles DE, Roffwarg HP, Rush AJ, Guzick DS (1990b). Age-adjusted threshold values for reduced REM latency in unipolar depression using ROC analysis. Biol Psychiatry 27: 841-853.

Goodyer IM, Herbert J, Tamplin A, Altham PME (2000). Recent life events, cortisol, dehydroepiandrosterone and the onset of major depression in high-risk adolescents. $\mathrm{Br} J$ Psychiatry 177: 499-504.

Goodyer IM, Park RJ, Herbert J (2001). Psychosocial and endocrine features of chronic first-episode major depression in 8-16 year olds. Biol Psychiatry 50: 351-357.

Gottesman II, Gould TD (2003). The endophenotype concept in psychiatry: etymology and strategic intentions. Am J Psychiatry 160: 636-645.

Halligan SL, Herbert J, Goodyer I, Murray L (2007). Disturbances in morning cortisol secretion in association with maternal postnatal depression predict subsequent depressive symptomatology in adolescents. Biol Psychiatry 62: 40-46.

Halligan SL, Herbert J, Goodyer IM, Murray L (2004). Exposure to postnatal depression predicts elevated cortisol in adolescent offspring. Biol Psychiatry 55: 376-381.

Hamilton M (1960). A rating scale for depression. J Neurol Neurosurg Psychiatry 23: 56-62.

Hammen C, Shih JH, Brennan PA (2004). Intergenerational transmission of depression: test of an interpersonal stress model in a community sample. J Consult Clin Psychol 72: 511-522.

Harris TO, Borsanyi S, Messari S, Stanford K, Cleary SE, Shiers HM et al (2000). Morning cortisol as a risk factor for subsequent major depressive disorder in adult women. Br J Psychiatry 177: 505-510.

Hollingshead AB (1975). Four Factor Index of Social Status. Department of Sociology, Yale University: New Haven, CT.

Holsboer F (2001). Stress, hypercortisolism and corticosteroid receptors in depression: implications for therapy. J Affect Disord 62: 77-91.

Ising M, Lauer CJ, Holsboer F, Modell S (2005). The Munich vulnerability study on affective disorders: premorbid neuroendocrine profile of affected high-risk probands. J Psychiatr Res 39: 21-28.

Ivanenko A, Johnson K (2008). Sleep disturbances in children with psychiatric disorders. Sem Pediatr Neurol 15: 70-78.

Joiner TE (2006). The Interpersonal, Cognitive, and Social Nature of Depression. Lawrence Erilbaum Associates: Mahwah, New Jersey.

Kaufman J, Birmaher B, Brent D, Rao U, Flynn C, Moreci P et al (1997). Schedule for Affective Disorders and Schizophrenia for School-aged Children-Present and Lifetime Version (K-SADSPL): initial reliability and validity data. J Am Acad Child Adolesc Psychiatry 36: 980-988.

Kaufman J, Martin A, King RA, Charney D (2001). Are child-, adolescent-, and adult-onset depression one and the same disorder? Biol Psychiatry 49: 980-1001.

Kendler KS, Gardner CO, Neale MC, Prescott CA (2001). Genetic risk factors for major depression in men and women: similar or different heritabilities and same or partly distinct genes? Psychol Med 31: 605-616.

Kessler RC, Avenevoli S, Merikangas KR (2001). Mood disorders in children and adolescents: an epidemiologic perspective. Biol Psychiatry 49: 1002-1014.

Kimura M, Winkelmann J (2007). Genetics of sleep and sleep disorders. Cell Mol Life Sci 64: 1171-1173.

Kirschbaum C, Wust S, Faig H-G, Hellhammer DH (1992). Heritability of cortisol responses to human corticotrophin- 
releasing hormone, ergometry, and psychological stress in humans. J Clin Endocrinol Metab 75: 1526-1530.

Kupfer DJ, Foster G, Reich L, Thompson KS, Weiss B (1976). EEG sleep changes as predictors in depression. Am J Psychiatry 133: 622-626.

Lauer C, Schreiber W, Holsboer F, Krieg J-C (1995). In quest of identifying vulnerabilitiy markers for psychiatric disorders by all-night polysomnography. Arch Gen Psychiatry 52: 145-153.

Lauer CJ, Bronisch T, Kainz M, Schreiber W, Holsboer F (1997). Pre-morbid psychometric profile of subjects at high familial risk for affective disorder. Psychol Med 27: 355-362.

Lauer CJ, Modell S, Schreiber W, Krieg J-C, Holsboer F (2004). Prediction of the development of a first major depressive episode with a rapid eye movement sleep induction test using the cholinergic agonist RS86. J Clin Psychopharmacol 24: 356-357.

Mannie ZN, Harmer CJ, Cowen PJ (2007). Increased waking salivary cortisol levels in young people at familial risk for depression. Am J Psychiatry 164: 617-621.

Mathew SJ, Coplan JD, Goetz RR, Feder A, Greenwald S, Dahl RE et al (2003). Differentiating depressed adolescent $24 \mathrm{~h}$ cortisol secretion in light of their adult clinical outcome. Neuropsychopharmacology 28: 1336-1343.

Meikle AW, Stringham JD, Woodward MG, Bishop DT (1988). Heritability of variation of plasma cortisol levels. Metab 37: 514-517.

Modell S, Ising M, Holsboer F, Lauer CJ (2005). The Munich vulnerability study on affective disorders: premorbid polysomnographic profile of affected high-risk probands. Biol Psychiatry 58: 694-699.

Modell S, Lauer CJ, Schreiber W, Huber J, Kreig J-C, Holsboer F (1998). Hormonal response pattern in the combined DEX/CRH test is stable over time in subjects at high familial risk for affective disorders. Neuropsychopharmacology 18: 253-262.

Monroe SM, Harkness K, Simons AD, Thase ME (2001). Life stress and the symptoms of major depression. J Nerv Ment Dis 189: $168-175$.

Monroe SM, Thase ME, Simons AD (1992). Social factors and the psychobiology of depression: relations between life stress and rapid eye movement sleep latency. J Abnorm Psychol 101: 528-537.

Morehouse RL, Kusumakar V, Kutcher SP, LeBlanc J, Armitage R (2002). Temporal coherence in ultradian sleep EEG rhythms in a never-depressed, high-risk cohort of female adolescents. Biol Psychiatry 51: 446-456.

Ouellet-Morin I, Boivin M, Dionne G, Lupien SJ, Arsenault L, Barr RG et al (2008). Variations in heritability of cortisol reactivity to stress as a function of early familial adversity among 19-monthold twins. Arch Gen Psychiatry 65: 211-218.

Perlis ML, Giles DE, Buysse DJ, Thase ME, Tu X, Kupfer DJ (1997). Which depressive symptoms are related to which sleep electroencephalographic variables? Biol Psychiatry 15: 904-913.

Perlis ML, Giles DE, Fleming G, Drummond SPA, James SP (1995). Sustained facial muscle activity during REM sleep in depressed subjects and its correlations with self-reported depression. J Affect Disord 35: 163-171.

Poland RE, McCracken JT, Lutchmansingh P, Lesser IM, Tondo L, Edwards C et al (1997). Differential response of rapid eye movement sleep to cholinergic blockade by scopolamine in currently depressed, remitted and normal control subjects. Biol Psychiatry 41: 929-938.

Poland RE, McCracken JT, Lutchmansingh P, Tondo L (1992). Relationship between REM sleep latency and nocturnal cortisol concentrations in depressed patients. J Sleep Res 1: 54-57.

Poland RE, Rubin RT (1982). Saliva cortisol levels following dexamethasone administration in endogenously depressed patients. Life Sci 30: 177-181.
Rao U (2006). Development and natural history of pediatric depression: treatment implications. Clin Neuropsychiatry 3: 194-204.

Rao U, Chen L-A (2009). Characteristics, correlates and outcomes of childhood and adolescent depressive disorders. Dialogues Clin Neurosci 11: 91-108.

Rao U, Dahl RE, Ryan ND, Birmaher B, Williamson DE, Giles DE et al (1996). The relationship between longitudinal clinical course and sleep and cortisol changes in adolescent depression. Biol Psychiatry 40: 474-484.

Rao U, McCracken JT, Lutchmansingh P, Edwards C, Poland RE (1997). Electroencephalographic sleep and urinary free cortisol in adolescent depression: a preliminary report of changes from episode to recovery. Biol Psychiatry 41: 369-373.

Rao U, Poland RE (2008). EEG sleep and HPA changes from episode to recovery in depressed adolescents. J Child Adolesc Psychopharmacol 18: 607-613.

Rechtschaffen A, Kales A (1968). A Manual of Standardized Terminology, Techniques, and Scoring System for Sleep Stages of Human Subjects. National Institutes of Health: Bethesda, MD.

Robert JJ, Hoffmann RF, Emslie GJ, Hughes C, Rintelmann J, Moore J et al (2006). Sex and age differences in sleep macroarchitecture in childhood and adolescent depression. Sleep 29: 351-358.

Rush AJ, Thase ME, Dubé S (2003). Research issues in the study of difficult to treat depression. Biol Psychiatry 53: 743-753.

Schmid DA, Brunner H, Lauer CJ, Uhr M, Yassouridis A, Holsboer $\mathrm{F}$ et al (2008). Acute cortisol administration increases sleep depth and growth hormone release in patients with major depression. J Psychiatr Res 42: 991-999.

Schreiber W, Lauer CJ, Krumrey K, Holsboer F, Krieg J-C (1992). Cholinergic REM sleep induction test in subjects at high risk for psychiatric disorders. Biol Psychiatry 32: 79-90.

Shaffer D, Gould MS, Brasic J, Ambrosini P, Fisher P, Bird H et al (1983). A Children's Global Assessment Scale (C-GAS). Arch Gen Psychiatry 40: 1228-1231.

Shapiro R, Keller M (1979). Longitudinal Interval Follow-Up Evaluation (LIFE). Massachusetts General Hospital: Boston, MA.

Shapiro SS, Wilk MB (1965). An analysis of variance test for normality (complete samples). Biometrika 52: 591-611.

Simons AD, Gordon JS, Monroe SM, Thase ME (1995). Toward an integration of psychologic, social, and biologic factors in depression: effects of outcome and course of cognitive therapy. J Consult Clin Psychol 63: 369-377.

Sitaram N, Dube S, Keshavan M, Davies A, Reynal P (1987). The association of supersensitivity cholinergic REM-induction and affective illness within pedigrees. J Psychiatr Res 21: 487-497.

Steiger A (2007). Neurochemical regulation of sleep. J Psychiatr Res 41: $537-552$

Thase ME, Kupfer DJ, Buysse DJ, Frank E, Simons AD, McEachran $\mathrm{AB}$ et al (1995). Electroencephalographic sleep profiles in single episode and recurrent unipolar forms of major depression: I. Comparison during acute depressive states. Biol Psychiatry 38: 506-515.

Thompson WD, Orvaschel H, Prusoff BA, Kidd KK (1982). An evaluation of the family history method for ascertaining psychiatric disorders. Arch Gen Psychiatry 39: 53-58.

Tsuno N, Besset A, Ritchie K (2005). Sleep and depression. J Clin Psychiatry 66: 1254-1269.

Uher R (2008). The implications of gene-environment interactions in depression: will cause inform cure?. Mol Psychiatry 13: 1070-1078.

Young AH (2004). Cortisol in mood disorders. Stress 7: 205-208.

Zalsman G, Oquendo MA, Greenhill L, Goldberg PH, Kamali M, Martin A et al (2006). Neurobiology of depression in children and adolescents. Child Adolesc Psychiatr Clin N Am 15: 843-868, vii-viii. 